\title{
Genre, territoire et promotion de la santé communautaire : enjeux théoriques et méthodologiques
}

Sara Aguirre Sánchez-Beato

\section{(2) OpenEdition}

\section{Journals}

Édition électronique

URL : http://journals.openedition.org/rfst/413

DOI : 10.4000/rfst.413

ISSN : 2492-3672

Éditeur

Espaces et SOciétés (UMR 6590)

Référence électronique

Sara Aguirre Sánchez-Beato, «Genre, territoire et promotion de la santé communautaire : enjeux théoriques et méthodologiques », Revue francophone sur la santé et les territoires [En ligne], Genre, territoire et santé, mis en ligne le 21 décembre 2015, consulté le 06 avril 2021. URL : http:// journals.openedition.org/rfst/413; DOI : https://doi.org/10.4000/rfst.413

Ce document a été généré automatiquement le 6 avril 2021.

La Revue francophone sur la santé et les territoires est mise à disposition selon les termes de la Licence Creative Commons Attribution - Pas d'Utilisation Commerciale - Partage dans les Mêmes Conditions 4.0 International. 


\title{
Genre, territoire et promotion de la santé communautaire : enjeux théoriques et méthodologiques
}

\author{
Sara Aguirre Sánchez-Beato
}

\section{NOTE DE L'ÉDITEUR}

Avec la collaboration de : Céline Mahieu [contributeur], Professeure, Université libre de Bruxelles - ULB, BelgiqueIsabelle Godin [contributeur], Professeure, Université libre de Bruxelles - ULB, BelgiqueLucía Gómez Sánchez [contributeur], Professeure, Universitat de Valencià - UV, Espagne Annalisa Casini [contributeur], Professeure, Université libre de Bruxelles - ULB, Belgique, Université catholique de Louvain - UCL, Belgique.

\section{Introduction et objectifs}

1 La proposition théorique et méthodologique que nous présentons est au fondement de notre projet de recherche mené au Centre de recherche Approches sociales de la santé de l'Université libre de Bruxelles sur la capacité des jeunes d'agir pour leur santé dans un cadre de promotion de la santé communautaire. À partir des enjeux théoriques et méthodologiques que nous décrivons dans cet article, nous souhaitons contribuer à la réflexion et au développement de nouvelles recherches empiriques qui abordent l'imbrication genre-territoire-santé dans le domaine de la promotion de la santé.

2 La période entre 18-24 ans, moment de transition entre l'adolescence et la vie adulte, correspond à un moment clé du développement personnel et social. En Région de Bruxelles-capitale une proportion importante des jeunes dans cette tranche d'âge se trouve dans une situation de précarité, des jeunes particulièrement vulnérables par rapport à l'insertion dans le marché de l'emploi et du logement, à l'enseignement, à la santé et au risque de pauvreté - un nombre croissant de jeunes demandant l'aide du 
CPAS ${ }^{1}$ (Observatoire de la santé et du social de Bruxelles, 2013). Selon un rapport de l'observatoire de la santé et du social de Bruxelles (Observatoire de la santé et du social de Bruxelles, 2013), les inégalités sociales de santé sont aussi présentes dans cette population, notamment en relation à la mortalité et à la santé perçue entre les filles et les garçons, la souffrance psychologique, l'accès aux soins et les habitudes de « soin de soi ». De par ces constats, notre projet de recherche se focalise sur la promotion de la santé et du bien-être des jeunes adultes dans la Région de Bruxelles-capitale.

3 La promotion de la santé, dont l'objectif est d'améliorer la santé et le bien-être et de réduire les risques de problèmes de santé, vise à donner aux populations les moyens d'assurer un plus grand contrôle sur leur propre santé (OMS, 1986; OMS, 2009). Ce champ de la santé publique conçoit la santé comme un «état de complet bien-être physique, mental et social » telle que définie par l'OMS (1986). Dans ce cadre, notre projet de recherche vise à étudier comment les inégalités sociales basées sur le genre, l'ethnicité, le statut migratoire, le statut socioéconomique, etc. conditionnent la capacité des jeunes d'agir pour leur santé, définie comme "la capacité des individus d'atteindre les objectifs de santé qu'ils poursuivent et d'agir pour leur propre santé" (Prah Ruger, 2010).

4 Ainsi, nous voudrions (a) cerner les différentes formes que prennent la capacité des jeunes d'agir pour leur santé et leur bien-être et à (b) analyser comment cette capacité est conditionnée par les inégalités sociales afin d' (c) identifier des pistes d'action pour améliorer l' empowerment des jeunes.

\section{L'imbrication genre-santé-territoire : perspectives théoriques}

\section{La capacité d'agir se façonne dans l'imbrication des inégalités sociales}

5 En mettant l'accent sur la maîtrise des populations sur leur propre santé, la promotion de la santé se focalise sur le rôle des personnes en tant qu'acteurs de leur santé, tout en reconnaissant l'importance de la situation sociale, économique et environnementale (Abel et Frohlich, 2011). Il est alors important de questionner la conceptualisation de ce rôle.

6 Comme Abel et Fröhlich (2011) l'avancent, dans certains programmes de santé on peut constater une tendance à assimiler les individus à leurs comportements, c'est-à-dire, à les considérer comme des porteurs de risques. Cette objectification des individus peut être identifiée dans bon nombre des initiatives adressées "aux fumeurs » ou "aux toxicomanes " pour citer quelques exemples, où les individus sont définis à partir de leurs comportements. Cette approche tend à individualiser les comportements de santé et à les conceptualiser comme de simples décisions rationnelles dans une logique de coût-bénéfice (Horrocks et Johnson, 2014). Elle s'accompagne fréquemment d'une rhétorique restrictive de l'éducation à la santé qui vise à enseigner aux individus à être plus sains ou à se protéger davantage, et tend par conséquent à sous-estimer tant les facteurs structurels contraignants que la capacité d'agir des acteurs dans les limites de ces contraintes (Abel et Frohlich, 2011). 
7 Dans la perspective que nous adoptons ici, les individus sont, par contre, considérés comme des êtres socialement situés, engagés et actifs dans le maintien de leur propre santé, en même temps qu'ils rencontrent des obstacles et des difficultés (Horrocks et Johnson, 2014).

8 Afin de rendre compte de cette vision nous mobilisons ici la notion de capacités capabilities - d'Amartya Sen (1993) car cette approche permet de centrer l'attention sur ce que les personnes sont efficacement capables de faire et d'être. Dans ce cadre, Sen (1993) distingue entre les fonctionnements ( functionings - les réalisations) et les capacités ( capabilities - la capacité de réaliser quelque chose). L'accent sur cette distinction découle de la vision que Sen a du bien-être lequel doit, selon lui, inclure non seulement les réalisations, mais aussi la capacité que les individus ont de choisir parmi un ensemble de réalisations différentes, c'est-à-dire, les opportunités que les personnes ont de réaliser leurs projets de vie. L'éventail de choix ainsi que la capacité de l'individu de choisir créent les conditions pour agir en faveur de sa santé. De plus, ces capacités peuvent être également des moyens pour une action transformatrice des structures sociales. Les stratégies de la promotion de la santé (OMS, 1986) se situent dans cette même lignée. Parmi ces stratégies, l' empowerment - « le processus d'aide à la réalisation de ce qui est en puissance chez un individu ou une communauté » (Saïas, 2009) entraîne la promotion de ressources, le développement de réseaux sociaux et la participation communautaire.

9 Ainsi nous considérons que la capacité des jeunes d'agir de façon favorable à leur santé et leur bien-être s'inscrit dans l'imbrication d'inégalités sociales qui donnent lieu à des inégalités sociales de santé (ISS). Les ISS sont « des injustices évitables en matière de santé (...) [qui sont] le reflet des inégalités que l'on peut constater en général au sein d'une société »(OMS, 2009).

\section{L'approche intersectionnelle : la conceptualisation des différences et leur identification par le territoire}

10 Penser aux inégalités sociales nous mène à réfléchir la conceptualisation de la différence et de la diversité au sein de la population. Nous pensons que la question de la diversité, qui met l'accent sur les différences au sein des populations, ne devrait pas être réduite à des questions culturelles mais elle devrait plutôt être analysée dans un cadre plus vaste d'inégalités structurelles. Ainsi, la conceptualisation de ces différences devient un aspect central dans la compréhension des inégalités sociales de santé.

11 Viruell-Fuentes et al (2012) dénoncent le fait que les catégories sociales sont souvent traitées comme de simples variables démographiques et réclament un changement conceptuel dans la façon dont on les conçoit afin d'adresser les rapports inégaux qui les sous-tendent. En même temps, tandis que les théories de la stratification sociale tendent à réduire la réalité à des groupes de population homogènes, la revue de littérature des études empiriques portant sur les inégalités en santé menée par Iyer et collaborateurs (2010) met en évidence le manque d'exactitudes des analyses lorsqu'une seule catégorie sociale est considérée. Cela montre que les groupes sociaux (hommesfemmes, riches-pauvres) ne sont pas homogènes tout en révélant la complexité des mécanismes producteurs d'inégalités de santé. 
12 En ce sens, le paradigme intersectionnel offre à la santé publique un cadre analytique et interprétatif pour repenser la conceptualisation, la recherche et l'analyse des inégalités sociales de santé (Bowleg, 2012).

13 La notion d'intersectionnalité, introduite par Kimberlee Crenshaw (1993) et issue des féminismes noirs, chicanos et postcoloniaux, est une approche qui s'est développée au niveau académique dans le champ des études de genre depuis les années 1990. Elle naît de la nécessité d'appréhender la diversité des expériences des femmes et des hommes, en raison d'autres rapports sociaux qui interagissent avec le genre, rendant visible parlà la nature mutuellement constitutive des inégalités sociales (Crenshaw, 1993; Anzaldúa, 1987 ; Hill Collins, 1990). Ainsi, selon cette approche il n'est pas suffisant de considérer les catégories sociales dans une logique additive, c'est-à-dire comme donnant lieu à une "somme de discriminations". Au contraire, la perspective intersectionnelle pointe l'importance de considérer en même temps différents rapports sociaux et de se centrer sur l'imbrication de ceux-ci car c'est bien cette interaction qui donne lieu à des expériences individuelles substantiellement différentes.

Depuis peu, l'approche intersectionnelle commence à être progressivement incorporée dans les recherches en santé publique, notamment au Canada et aux États-Unis (Schulz et Mullings, 2006 ; Iyer et al., 2010 ; Morgen, 2010 ; Rogers et Kelly, 2011 ; Bowleg, 2012 ; Hankivsky et al., 2012). Par exemple, face à l'augmentation des taux d'infection par le VIH auprès des femmes en situation vulnérable à Ontario (Canada), la recherche intersectionnelle de Logie et al. (2011) montre l'interdépendance et la constitution mutuelle des rapports sociaux et des inégalités - comme le sexisme, le racisme, le stigma associé au VIH et au travail du sexe, l'homophobie et la transphobie - en tant qu'obstacles dans le bien-être et la santé des femmes séropositives ainsi que dans leurs stratégies de coping avec la maladie. Une autre expérience intéressante dans le domaine de la santé publique est le cadre théorique développé par Hankivsky et al. (2012) pour l'analyse de politiques de santé avec une approche intersectionnelle. Par contre, le potentiel de cette approche pour réduire les inégalités sociales demeure encore peu exploité, d'où la nécessité de développer plus de recherches fondées empiriquement (Hankivsky et Christoffersen, 2008).

15 L'intersectionnalité correspond à un véritable changement de paradigme qui se caractérise par certains principes-clés (Bowleg, 2012 ; Christensen et Qvotrup, 2012). L'accent est ainsi particulièrement mis sur le pouvoir dans les rapports sociaux, sur la constitution mutuelle de ces rapports (principe de la non-addition), ainsi que sur la compréhension des subjectivités comme multiples, croisées et dépendantes du contexte. Ainsi, la sélection des catégories sociales à étudier est une «question empirique ouverte » (Hancock, 2007, p. 251).

16 En respectant ces principes, notre projet ne s'intéresse pas à des groupes spécifiques des jeunes (par exemple, « les femmes », « les personnes migrantes », etc.) mais plutôt aux rapports sociaux (genre, classe, ethnicité) présents dans l'ensemble des jeunes. Au lieu de sélectionner a priori quelques groupes, il nous semble nécessaire d'adopter une démarche inductive afin que ces rapports sociaux complexes et pertinents pour notre questionnement, puissent émerger du contexte spécifique choisi. Cette démarche nous permet aussi d'éviter une " approche basée sur les minorités " ${ }^{\mathrm{i}}$ (Giolla Easpaig et al., 2013) dans laquelle une dimension de l'identité est privilégiée comme la caractéristique qui nous permet de comprendre un groupe particulier de personnes (par exemple, la « communauté gay»). Selon ces auteurs (Giolla Easpaig et al., 2013) cette approche 
identitaire implique de risques importants : elle fixe et valide certaines dimensions sur lesquelles "les autres » sont différenciés de "la majorité », en même temps que son homogénéité est présumée; et elle risque de normaliser les pratiques de groupes dominants et privilégiés. En plus, comme Bowleg (2012) l'affirme dans son analyse de programmes de santé publique, lorsque l'on sélectionne des groupes spécifiques, par exemple "les femmes et les migrants", on aborde implicitement ces populations comme étant mutuellement exclusives.

17 Ainsi, une approche territoriale de communauté évite la stigmatisation de groupes spécifiques de la population et nous permet d'appréhender l'imbrication de rapports sociaux. Il s'agit donc de partir de l'ensemble de la communauté d'un territoire défini et délimité afin d'analyser les dynamiques de pouvoir dans leur ensemble (Christensen et Qvotrup, 2012).

Comme Giblin (2011) l'affirme, plus le territoire est vaste plus les inégalités de santé sont masquées. Ainsi, nous considérons que l'échelle locale, et plus précisément celle de la commune, est l'échelle d'analyse la plus pertinente pour notre étude. La Région Bruxelles-capitale est composée de 19 communes, 118 quartiers et 724 secteurs statistiques (Observatoire de la Santé et du Social Bruxelles-Capitale, 2015) (voir Figure 1).

19 En Belgique, la commune est «le pouvoir le plus proche du citoyen " (Région de Bruxelles-capitale, 2015), ayant des missions comme le registre de la population, les activités sociales (y compris les actions de promotion et prévention en matière sanitaire), les activités culturelles (sportives, éducation permanente, animation culturelle), le logement, l'adoption d'instruments de planification et d'urbanisme (salubrité) (AVCB $\left.{ }^{2}, 2007\right)$. Une coordination de l'égalité de chances au niveau local soutient les communes dans cet aspect (Région de Bruxelles-capitale, 2015).

Cependant, en raison de différences socioéconomiques et sociodémographiques au sein des communes et des caractéristiques de la promotion de la santé communautaire (actions de proximité, participation), l'échelle d'analyse de notre étude est au niveau des quartiers. Les communes de la Région bruxelloise étant des ensembles très hétérogènes et de grande dimension, un découpage administratif infra-communal a été réalisé en 2007 par l'Institut Bruxellois de Statistique et d'Analyse (IBSA) afin d'« appréhender la complexité de l'espace urbain» (IBSA, 2007, p. 2). C'est suite à un projet de découpage optimal, correspondant à la réalité de terrain, que les quartiers, décrits comme «l'espace de vie au sein duquel s'articule une part non négligeable des activités quotidiennes et auquel les habitants s'identifient », ont été définis (IBSA, 2007, p.2). En contrepoint des découpages plus classiques basés sur l'homogénéité socioéconomique des habitants, ce projet (IBSA, 2007) a mis l'accent sur le milieu de vie commun et sur le sentiment d'appartenance. Cette unité territoriale, idéale du point de vue de la taille géographique et de la population pour promouvoir la santé communautaire, a en plus été construite sur la base d'un ressenti partagé, incluant le point de vue des habitants. 




Source : Observatoire de la santé et du social de Bruxelles-capitale, 2015

\section{La capacité d'agir comme processus résultant de l'interaction entre le corps, les ressources individuelles et les ressources du quartier}

21 Se différenciant de certaines approches épidémiologiques dont l'attention est portée sur la description et la quantification des inégalités sociales de santé en relation aux comportements, aux risques, à la morbidité et à la mortalité, notre projet de recherche s'intéresse plutôt aux processus qui mènent à ces inégalités. Dans cette lignée, Fröhlich et Abel (2014) proposent de prêter attention à l'interaction des facteurs structurels (ressources individuelles et ressources des quartiers) et de différentes capacités des personnes d'agir pour leur santé.

22 Comme Staunaes (2003) l'affirme, il s'agit de surmonter la tendance à percevoir les sujets comme étant déterminés par les systèmes sociaux, étant donné que cela rend difficile la compréhension des subversions, des ambigüités et des exceptions au niveau des sujets. L'un des principes-clés en psychologie communautaire de la santé est le principe de "personne dans le contexte ", qui envisage les problèmes comme étant le produit de l'interaction des personnes avec un environnement social déterminé, ce qui s'inscrit dans un binarisme «structure - capacité d'agir » (Giolla Easpaig et al., 2014). Par contre, cette interaction devrait être comprise comme un processus de constitution mutuelle plutôt que comme un déterminisme cause-effet unilatéral (Cornish, 2004).

Ainsi, les actions des jeunes qui ont un impact sur la santé seront influencées, mais pas déterminées, par des systèmes sociaux inégaux étant donné la capacité des jeunes d'agir. Nous proposons d'analyser cette capacité par rapport à trois éléments clé pour 
le maintien de la santé et autour desquels se configurent les inégalités sociales: le corps, les ressources individuelles et les ressources du quartier.

\section{Le corps}

24 Comme Mason (2013) l'affirme, «le corps et les inégalités sociales se constituent mutuellement ». La notion d' embodiment ( incarnation, en français) vise à surmonter les dichotomies « corps-esprit » et " nature-culture » (Pujol et al., 2003). Cette perspective s'oppose ainsi à la compréhension scindée de l'être humain : d'un côté la pensée et de l'autre, le corps; une compréhension où la corporéité est réduite à la chair, reléguée au rôle d'objet de connaissance, jamais considérée comme sujet (Pujol et al., 2003).

25 Ces clivages se retrouvent aujourd'hui dans la division entre le sexe, en tant que «réalité biologique " - et par conséquent, objective, naturelle et immuable - et le genre , comme "construction sociale» de rôles et comportements féminins et masculins - et dès lors, culturelle et modifiable (Haraway, 1988).

Le modèle sexe/genre, largement adopté par le féminisme institutionnel et les organismes internationaux, a été présenté comme la solution au débat sur la différence sexuelle et l'origine des discriminations, mais il entraîne des effets pervers: l'immutabilité du sexe implique qu'il existe une réalité objective clairement définie (le sexe masculin et le sexe féminin), impossible de modifier, qui s'impose à la réalité sociale (Delphy, 2001) et dont toute variation est interprétée comme une déviance (par exemple, les personnes intersexuées). C'est une approche qui à notre avis échoue à prendre en compte la construction sociale du corps et la participation de celui-ci à la formation du savoir et des pratiques.

Comme l'exprime l'idée de performativité (Butler, 1990), le genre, mais aussi le corps sexué sont des produits culturels qui sont devenus naturels à force de répétitions, mais la matérialité des corps participe aussi à sa construction et les subjectivités qui en résultent constituent une expérience incarnée pour les sujets.

Cette approche peut être appliquée non seulement à l'étude de la dimension genrée du corps, mais aussi de sa dimension ethnicisée, âgée, handicapée, etc., ainsi qu'à leur interaction. Ainsi, les catégories sociales basées sur la classification de signes corporels sont structurées et maintenues socialement à travers de pratiques et des actions dans la vie quotidienne.

29 Pour citer quelques exemples, le domaine de l'alimentation et de la pratique sportive est très illustrateur. De ce point de vue, les régimes diététiques représentent non seulement une influence des normes du genre sur le corps mais aussi une construction du genre en soi, de ce que c'est ou doit être " une femme ", à travers cette action. Cette construction n'est pas seulement en relation avec le genre, mais avec l'interaction de celui-ci avec d'autres catégories sociales, comme l'ethnicité et la classe sociale. Par exemple, la construction de ce que doit être "une femme » varie en fonction des rapports ethniques et de classe. Il en va de même de la pratique sportive intensive et de la consommation d'anabolisants censée développer une musculature qui renvoie à une certaine image idéalisée du corps masculin, traversée elle aussi par des stéréotypes ethniques et de classe sociale. 


\section{Ressources individuelles}

30 Abel et Frohlich (2011) proposent un modèle centré sur l'individu actif, permettant d'expliquer non seulement la (re)production d'inégalités sociales en santé, mais aussi leur potentielle modification. L'idée centrale est que les inégalités de santé ne peuvent pas uniquement être expliquées par l'inégale distribution des ressources individuelles pour la santé (capitaux économique, social et culturel), mais qu'il y a aussi « une distribution inégale des chances et des capacités des personnes à atteindre les différentes formes de capital constamment appuyées et complétées les unes par les autres pour que leur interaction ait un résultat positif pour la santé » (Abel et Frohlich, 2011, p. 238). Ainsi, la mobilisation des différents types de ressources et leur combinaison au bénéfice de la santé requièrent des individus actifs . La capacité d'agir de ceux-ci joue un rôle important dans la transformation des inégalités sociales en inégalités en santé.

Or, l'action des sujets en vue de transformer un type de ressource en un autre est influencée par la capacité d'agir des personnes. Ainsi, la transformation du capital économique en, par exemple, un cours de cuisine saine (capital culturel) est une action où la capacité d'agir est conditionnée par les inégalités sociales basées sur le genre, l'ethnicité, le statut socioéconomique, etc. Dans ce cas, le public majoritairement féminin de ce type de cours répond à la catégorisation des femmes comme celles qui prennent soin des autres (à travers la cuisine) et contribue en même temps à la construction de cette catégorisation.

\section{Ressources du quartier}

Le quartier peut être pensé non seulement comme un espace géographique pour étudier la distribution de "risques" pour les personnes y résidant, mais aussi comme un des contextes sociaux offrant des ressources pour la santé de façon inéquitable (Fröhlich et Abel, 2014).

33 Le choix résidentiel est influencé par les ressources économiques et les préférences de style de vie ${ }^{3}$ des sujets ainsi que par les caractéristiques physiques et les ressources du quartier (Bernard et al., 2007). Ce choix contribue à son tour à construire le quartier.

Dès lors, d'une part, les catégories sociales sont distribuées de façon inégale dans le territoire à cause du processus décrit, d'autre part, au sein d'un même quartier, différentes catégories sociales ont un accès et une utilisation différentielle de ces ressources.

35 À titre d'illustration, une recherche sur les espaces de loisirs des jeunes a montré que la cours des écoles et l'espace public d'un quartier n'étaient pas utilisés de la même façon par les garçons et les filles (Maruejouls-Benoit, 2014). L'étude montre une occupation «sexuée » de la cours, les garçons dits «sportifs» se situant au centre, les filles et les groupes mixtes en périphérie (Maruejouls-Benoit, 2014). L'étude montre aussi que les activités sportives et culturelles proposées par les municipalités étaient pratiquées différemment par les filles (40\%) et les garçons (60\%) (Maruejouls-Benoit, 2014).

En même temps, les ressources du quartier peuvent être aussi modifiées par les actions des sujets, par exemple, quand les habitants s'organisent pour créer un jardin collectif ou pour demander des services publics. 
37 En conclusion, bien que le corps, les ressources individuelles et les ressources du quartier aient été présentés individuellement, ils doivent être conçus en interaction, faisant partie de microprocessus de la vie quotidienne qui définissent la capacité d'agir des jeunes par rapport à leur santé, question clé dans la compréhension des rapports sociaux inégaux (Giolla Easpaig et al., 2013).

\section{L'imbrication genre-santé-territoire : perspectives méthodologiques}

Notre projet de recherche prévoit de mener une étude empirique dans la commune de Forest, en Région de Bruxelles-capitale. Cette commune a été choisie en fonction d'un critère de diversité interne par rapport aux origines, au statut socioéconomique (SSE) de la population et au nombre élevé de jeunes (Observatoire de la santé et du social Bruxelles, 2010), ainsi que de l'existence d'acteurs publics et de la société civile intéressés à participer à l'étude. Du point de vue méthodologique, notre démarche s'appuie sur deux volets : un premier volet à visée exploratoire et un deuxième volet qualitatif d'analyse intersectionnelle proprement dite. Nous proposons aussi une approche comparative inter-quartiers afin de mieux comprendre la relation entre le quartier, la santé et le genre.

\section{La sélection de catégories sociales : méthode d'analyse en groupe (MAG)}

39 Comme nous l'avons précisé plus haut, l'approche intersectionnelle considère la sélection a priori de groupes spécifiques de la population faisant l'objet de l'étude comme étant une démarche méthodologique contestable. Certains chercheurs en intersectionnalité proposent d'adopter une approche géographique (Christensen et Qvotrup, 2012) et de se concentrer sur des domaines ou des problématiques concrètes où des inégalités sociales sont particulièrement présentes (Verloo, 2014). Ainsi, selon eux, il s'agirait de cibler un quartier particulier (ou plusieurs si l'on vise à réaliser des comparaisons inter-quartiers comme l'on suggère ci-dessous) et d'identifier et d'analyser les rapports sociaux y présents.

40 Cependant, le nombre de catégories sociales pouvant être très élevé, Christensen et Qvotrup (2012) suggèrent de partir d'un nombre limité de points d'ancrage , c'est-à-dire de catégories sociales particulièrement importantes en fonction du domaine choisi. Ce choix stratégique nous permet ainsi de rendre l'analyse gérable et de mettre l'accent sur les catégories sociales les plus importantes dans le contexte de notre recherche. Selon ces chercheurs (Christensen et Qvotrup, 2012), les catégories sociales découlent de l'expérience vécue des sujets. En fonction de cette dernière, le genre serait un des possibles points d'ancrage parmi d'autres.

41 Comme nous l'avons précisé, l'analyse intersectionnelle demande à être appliquée dans un domaine concret, dans notre cas la capacité des jeunes d'agir pour leur santé et leur bien-être dans un quartier déterminé. La logique inductive que nous proposons nécessite d'employer une technique d'enquête qui nous permette d'un côté, d'identifier des "points d'ancrage" comme le genre, le statut socioéconomique ou l'ethnicité, c'est-à-dire des rapports sociaux qui conditionnent la capacité d'agir des jeunes. D'un 
autre côté, ce volet vise à la réalisation d'un diagnostic de ressources et de contraintes des jeunes pour agir pour leur bien-être. Dans ce cadre, nous proposons de mobiliser la méthode d'analyse en groupe (MAG).

42 Encore méconnue, cette méthode se révèle idéale pour une première phase exploratoire dans la mesure où elle se base sur des expériences vécues des participants et se situe dans une perspective relationnelle (Van Campenhoudt et al, 2009). Il s'agit d'un « débat organisé de manière procédurale » où l'on vise à saisir la dynamique des relations sociales (Van Campenhoudt et al, 2009, p. 11). L'intérêt de cette méthode réside notamment en sa capacité de cerner les tensions et les divergences présentes dans le discours sur l'objet d'étude considéré. Comme ses promoteurs l'affirment, la MAG « vise précisément à articuler la prise en compte de la diversité d'expériences et à élaborer une analyse d'ensemble, permettant de reconstruire le système d'action duquel les participants sont partie prenante " (Van Campenhoudt et al, 2009, p. 3). En opposition à la méthode des focus groups dont l'homogénéité des participants est une condition essentielle, dans la MAG la composition des groupes est le plus souvent diversifiée (Van Campenhoudt et al, 2009).

La MAG nous permettra à la fois de réaliser un diagnostic des ressources et des contraintes des jeunes pour agir pour leur santé et leur bien-être et d'identifier nos points d'ancrage, c'est-à-dire, les catégories sociales qui seront considérées dans l'étude à partir du propre vécu des sujets.

\section{L'expérience vécue de l'intersectionnalité : récits de la vie quotidienne}

44 Bien que la MAG nous permette d'identifier les catégories sociales et les dynamiques d'un quartier, nous considérons qu'elle n'est pas adéquate pour saisir l'expérience de l'intersectionnalité telle qu'elle est vécue au niveau des sujets, pour la compréhension des interactions des catégories sociales, vécues de façon unitaire par les sujets, (re)produites et transgressées. C'est pour cette raison que nous proposons la combinaison avec d'autres méthodes.

45 Pour ce faire, et inspirées par l'étude empirique intersectionnelle de Christensen et Qvotrup Jensen (2012), nous proposons la technique des récits ${ }^{\text {ii }}:$ des histoires de la vie quotidienne. Dans cette technique, l'interaction du genre avec d'autres catégories sociales découle de la façon dont les personnes racontent leurs vies. Les thèmes des récits concernent les ressources et les contraintes identifiées dans le diagnostic réalisé à travers la MAG.

46 Les récits sont une façon importante d'approcher la construction des subjectivités dans leurs aspects symboliques, mais aussi dans leurs aspects matériels liés à la santé (le corps, les ressources individuelles, les ressources du quartier). Ainsi, l'analyse du discours réalisée avec une optique intersectionnelle dans l'étude, permettra de comprendre l'influence de ces aspects symboliques et matériels sur la capacité des jeunes d'agir pour leur santé.

\section{Différents quartiers, différente santé : approche comparative}

47 La relation entre le territoire et la santé est une question très débattue (Bernard et al., 2007 ; Galéa et Vlahov, 2005). Dans l'approche que nous adoptons, elle est présentée 
comme un processus complexe où les ressources du quartier sont une base matérielle pour les pratiques liées à la santé, en même temps qu'elles sont modifiées par celles-ci.

En raison de la distribution des catégories sociales entre les quartiers, ainsi que des différences dans les ressources disponibles, il nous semble intéressant d'adopter une approche comparative entre plusieurs quartiers.

À titre d'exemple, dans le cas de notre recherche en Région de Bruxelles-capitale (RBC), nous avons identifié les quartiers de la commune de Forest (Figure 2) comme territoires potentiels pour l'étude. Selon les statistiques régionales, les caractéristiques démographiques, socioéconomiques et les indicateurs de santé de la commune de Forest ne s'écartent pas de la moyenne régionale, avec une population relativement mixte. Néanmoins, ces statistiques mettent en évidence l'existence d'importantes inégalités entre l'Est et l'Ouest de la commune, séparés physiquement par des zones vertes (Observatoire de la santé et du sociale de Bruxelles, 2010). L'étude des variations intra-communales est ainsi cruciale pour comprendre les territoires concernés, une logique qui s'applique à la plupart des communes de la Région Bruxelles-capitale. Ainsi, le choix des deux quartiers sera basé sur la fragmentation sociale que présente la commune concernée, avec des quartiers à l'Ouest hébergeant une population relativement favorisée et des quartiers à l'Est situés à côté des voies ferrées accueillant une population plus précarisée et plus diverse du point de vue de l'origine ethnique.

Figure 2 : Carte de la commune de Forest



Source : Observatoire de la santé et du social de Bruxelles-capitale, 2010

À partir de l'identification des ressources et des contraintes des jeunes pour agir pour leur santé dans les deux quartiers choisis, l'analyse de cette capacité d'agir dans chaque quartier permettra d'identifier non seulement les différentes ressources des quartiers, mais aussi la différente utilisation de ces ressources en fonction des diverses catégories sociales présentes dans les quartiers. 
51 L'objectif de la comparaison de deux ou plusieurs quartiers ne serait pas la constatation de faits, mais l'identification et la comparaison des processus différents et, en conséquence, de possibles pistes d'action pour la promotion de la santé.

\section{Conclusion}

Face à certaines tendances en promotion de la santé qui conceptualisent les actions liées à la santé comme une question individuelle ou de libre choix, nous suggérons de considérer les individus comme ayant un rôle actif dans le maintien de leur santé. Par contre, nous considérons que cette capacité d'agir se voit influencée par des catégories sociales (rapports de genre, d'ethnicité, de classe, etc.). Cet article présente une perspective intersectionnelle pour la compréhension du genre en tant que facteur qui influence la santé, c'est-à-dire, en prenant compte de son interaction avec d'autres catégories sociales. Ainsi, nous proposons d'adresser les processus bidirectionnels entre la capacité d'agir et les facteurs structurels, qui inclut des aspects symboliques mais aussi matériels. En ce sens, nous suggérons l'analyse du corps, des ressources individuelles et des ressources du quartier.

53 Ainsi, avec cette perspective théorique, nous proposons une démarche méthodologique novatrice pour l'étude empirique de l'intersectionnalité dans le domaine de la promotion de la santé. Suivant une logique inductive, l'approche comparative et la méthodologie qualitative, ainsi que les techniques de la méthode d'analyse en groupe et les récits de vie, seraient des propositions méthodologiques susceptibles de dépasser les défis relevés par l'étude empirique de l'intersectionnalité dans ce domaine.

De notre point de vue, cette proposition théorique et méthodologique permettrait de mieux comprendre l'intersection des catégories sociales présentes dans un territoire spécifique et de les aborder dans leur complexité, en évitant des réponses qui simplifient la réalité sociale. En même temps, elle pourrait contribuer à comprendre les mécanismes qui expliquent comment les inégalités sociales se concrétisent en inégalités sociales de santé.

55 En outre, l'approche comparative proposée permettrait d'appréhender l'impact des quartiers et de leurs ressources sur la santé de la population. Cette perspective pourrait être utilisée également dans la comparaison entre villes différentes ou entre milieu urbain et milieu rural.

\section{BIBLIOGRAPHIE}

Abel, T. and Frohlich, K. L. (2011), Capitals and capabilities: Linking structure and agency to reduce health inequalities. Social science and medicine, 74, pp. 236-244.

Anzaldúa, G. (1987), Borderlands/La Frontera: The New Mestiza. Aunt Lute Books. 
AVCB - Association de la Ville et des Communes de la Région de Bruxelles-Capitale ASBL (2007), Les missions de la commune.

Bernard, P., Charafeddine, R., Frohlich, K.L., Daniel, M., Kestens, Y. and Potvin, L. (2007), Health inequalities and place: a theoretical conception of neighbourhood. Social Science and Medicine 65, pp. 1839-1852.

Bowleg, L. (2012), The problem with the phrase Women and Minorities: Intersectionality - an important theoretical framework for public health. American Journal of Public Health , 102 (7), pp. 1267-73.

Butler, J. (1990), Gender Trouble, Feminism and the Subversion of Identity . London: Routledge.

Cornish, F. (2004), Making 'context' concret : A dialogical approach to the society-health relation. Journal of Health Psychology 9 (2): 281-294.

Crenshaw, K. (1993), Mapping the Margins: Intersectionality, Identity Politics, and Violence against Women of Color. Standford Law Review , 43, pp. 1241-1299.

Christensen A-D. and Qvotrup Jensen, S. (2012), Doing Intersectional Analysis: Methodological Implications for Qualitative Research. NORA - Nordic Journal of Feminist and Gender Research ; 20(2): p. $109-125$.

Delphy, C. (2001). L'ennemi principal. Penser le genre . Paris: Syllepse.

Frohlich, K.L. and Abel,T. (2014), Environmental justice and health practices : understanding how health inequities arise at the local level. Sociology of Health and Illness, Vol. 36 (2), pp. 199-212.

Galea, S. and Vlahov, D. (2005), Urban Health: Evidence, Challenges, and Directions. Annual Review of Public Health , 26 (1), pp. 341-365.

Giblin, B. (2011), Santé publique et territoires. Hérodote , 4 n ${ }^{\circ} 143$, p. 3-12.

Giolla Easpaig, B.N., Fryer, D.M., Linn, S.E. and Humphrey, R.H. (2013), A queer-theoretical approach to community health psychology. Journal of Health Psychology, Vol. 19 (1), pp. 117-125. Hancock, A-M (2007), Intersectionality as a normative and empirical paradigm. Politics \& Gender ; 3(2): p. 248-254.

Hankivsky, O. and Christoffersen, A. (2008), Intersectionality and the determinants of health: A Canadian perspective. Critical Public Health , 18 (3), p. 271-283.

Hankivsky, O. (2012), Women's health, men's health, and gender and health: Implications of intersectionality. Social Sciences and Medicine, 74, pp. 1712-1720.

Hankivsky, O. (Ed.). (2012). An Intersectionality-Based Policy Analysis Framework. Vancouver, BC: Institute for Intersectionality Research and Policy.

Haraway, D. (1988), Situated knowledges: The science question in feminism and the privilege of partial perspective. Feminist Studies , 14 (3), pp. 575-599.

Hill Collins, P (1990), Black Feminist Thought: Knowledge, Consciousness and the Politics of Empowerment. NY: Hyman.

Horrocks et Johnson (2014), A socially situated approach to inform ways to improve health and well-being. Sociology of Health and Illness , 20, pp. 1-12.

IBSA (2007), Conception d'un monitoring des quartiers couvrant l'ensemble du territoire de la Région de Bruxelles-Capitale . Bruxelles : Institut Bruxellois de Statistique et d'Analyse. 
Iyer A., Sen G. and Östlin, P. (2010), Inequalities and Intersections in Health. In Gender Equity in Health. The Shifting Frontiers of Evidence and Action , p. 70-95. New York: Routledge.

Logie CH, James L, Tharao W, Loutfy MR (2011) HIV, Gender, Race, Sexual Orientation, and Sex Work: A Qualitative Study of Intersectional Stigma Experienced by HIV-Positive Women in Ontario, Canada. PLoS Med 8(11): e1001124. doi:10.1371/journal.pmed.1001124

Maruejouls-Benoit, E. (2014), Mixité, égalité et genre dans les espaces du loisir des jeunes. Pertinence d'un paradigme féministe. Thèse de doctorat. Université Michel de Montaigne Bordeaux 3, France. URL : http://www.theses.fr/2014BOR30024

Mason, K. (2013), Social Stratification and the Body: Gender, Race and Class. Sociology Compass , 7/8, pp. 686-698.

Morgen S. (2010), Movement-grounded theory. Intersectional analysis of health inequities in the United States. In Gender Equity in Health. The Shifting Frontiers of Evidence and Action, p. 394-423. New York: Routledge.

Observatoire de la santé et du social Bruxelles-capitale (2015), Bruxelles en cartes. URL : http:// www.observatbru.be/documents/contexte-bruxellois/bruxelles-en-cartes.xml?lang=fr

Observatoire de la santé et du social de Bruxelles (2013), Jeunes en transition, adultes en devenir. Rapport bruxellois sur l'état de la pauvreté . Bruxelles.

Observatoire de la santé et du social Bruxelles-capitale (2010), Fiches communales d'analyse des statistiques locales en Région bruxelloise.

OMS (1986), Chartre d'Ottawa. Genève : Organisation mondiale de la Santé.

OMS (2009), Milestones in Health Promotion. Statements from Global Conferences . Gineva: World Health Organisation.

Prah Ruger, J. (2010), Health Capability: Conceptualization and Operationalization. American Journal of Public Health, Vol. 100, 1.

Pujol, J., Montenegro, M. y Balash, M. (2003), Los límites de la metáfora lingüística: implicaciones de una perspectiva corporeizada para la práctica investigadora e interventora. Política y sociedad, 40, pp. 57-70.

Région de Bruxelles-capitale (2015), Pouvoirs locaux . http://www.pouvoirslocaux.irisnet.be/fr

Rogers J. and Kelly U. A. (2011), Feminist intersectionality: Bringing social justice to health disparities research. Nursing Ethics , 18, pp. 397-407.

Saïas, T. (2009), Le cadre conceptuel de la psychologie communautaire. Dans Thomas Saïas (Ed.) Introduction à la psychologie communautaire. Paris : Dunod.

Schulz, A. J. and Mullings, L. (2006), Gender, race, class and health. Intersectional approaches . New York: Jossey Bass.

Sen, A. (1993), Capability and well-being. M. Nussbaum, A. Sen (Eds.), The quality of life, Clarendon Press, Oxford, pp. 30-53.

Staunaes, D (2003), Where have all the subjects gone? Bringing together the concepts of intersectionality and subjectification. NORA -Nordic Journal of Feminist and Gender Research; 11(2): p. 101-110.

Van Campenhoudt, L., Franssen A. et Cantelli F. (2009), La méthode d'analyse en groupe. Explication, applications et implications d'un nouveau dispositif de recherche. SociologieS . URL : http://sociologies.revues.org/2968 
Verloo, M. (2014), Doing intersectionality: dealing with the intersection of privilege and penalty. Presentation in Symposium Practices of Intersectionality , 27 March 2014, Radboud University Nijmegen, The Netherlands.

Viruell-Fuentes, E. A., Miranda, P. Y. and Abdulrahim, S. (2012), More than culture: Structural racism, intersectionality theory, and immigrant health. Social science and medicine. 75 , pp. 2099-2106.

\title{
NOTES
}

1. En Belgique, les CPAS, Centres Publics d'Action Sociale, sont les structures publiques qui assurent la prestation de services sociaux et veillent au bien-être de chaque citoyen et citoyenne disposant de moyens de subsistance insuffisants ou étant sans domicile fixe. Chaque commune ou ville a son propre CPAS offrant un large éventail de services tels que, entre autres, l'aide financière, l'aide au logement, l'aide médicale et psychosociale et l'assistance judiciaire.

2. Association de la Ville et des Communes de la Région de Bruxelles-Capitale.

3. Italiques ajoutées.

\section{NOTES DE FIN}

\author{
i. « Minoritising approach » en anglais - traduction propre. \\ ii. « Narratives » en anglais.
}

\section{RÉSUMÉS}

Dans une perspective de promotion de la santé, qui vise à donner aux personnes la maîtrise de leur propre santé, la proposition théorique et méthodologique que nous présentons s'intéresse à comprendre comment les inégalités sociales basées sur le genre, l'ethnicité, le statut socioéconomique, etc. conditionnent la capacité des jeunes d'agir pour leur santé. Nous focalisant sur les jeunes (18-24 ans), nous mobilisons la notion de «capacités » d'Amartya Sen (1993) pour concevoir le rôle actif des individus par rapport à leur santé, ainsi qu'une approche intersectionnelle qui, au lieu d'analyser les inégalités sociales de façon isolée, met l'accent sur l'imbrication de celles-ci afin de mieux comprendre les mécanismes qui les produisent. Nos objectifs sont (a) cerner les différentes formes de capacité d'agir qu'on les jeunes pour leur santé prend et à (b) analyser comment cette capacité est conditionnée par les inégalités sociales afin d' (c) identifier des pistes d'action pour améliorer l' empowerment des jeunes. Notre démarche méthodologique s'appuie sur deux volets. Un premier volet, à visée exploratoire, utilisera la méthode d'analyse en groupe afin de réaliser un diagnostic de ressources et de contraintes des jeunes pour agir pour leur santé, ainsi que d'identifier les catégories sociales les plus pertinentes pour l'analyse intersectionnelle. Sur cette base, le deuxième volet, basé sur la technique des 
récits de vie, a pour objectif de saisir l'interaction des inégalités sociales telle qu'elle est vécue par les jeunes et d'analyser comment cette interaction façonne leur capacité d'agir de façon favorable à leur santé. Ainsi, nous décrivons une démarche méthodologique pour l'étude de la capacité d'agir pour la santé afin de proposer une nouvelle grille de lecture, empiriquement fondée, pour la conceptualisation des programmes en promotion de la santé.

Health promotion is "the process of enabling people to increase control over, and to improve, their health" (OMS, 2009, p.1), putting the emphasis on the role of people as actors of their health. However, as Horrocks and Johnson (2014) claim, mainstream perspectives tend to conceptualize health-related actions as individually driven - hence the focus on lifestyles as a matter of choice. Drawing on these authors' critical perspective, we consider these actions as social practices that are historically and geographically situated. In this article we address the relation between social categories - such as gender, class and ethnicity - and health-related social practices. In this regard, we propose intersectionality (Crenshaw, 1993) as an approach to study the interaction of social categories.

In spite of the current interest that the intersectional approach has raised in the health field, its study has been mainly theoretical; therefore, more empirically-grounded research is needed. However, the empirical study of intersectionality is not unproblematic, its core principles raising a number of methodological challenges. In this article we present the theoretical and methodological proposal that conforms the core of our research about community health promotion in the Region of Brussels in order to address and empirically study the relation between gender, territory and health.

Drawing on post-structural approaches the first part of the article presents a theoretical perspective to understand the complex relation between social categories, territory and health through the notion of health-related social practices. In this perspective the territory is considered in two different ways: as a methodological tool and -together with the body and individual ressources- as an element of health-related social practices.

Community action being one of the main characteristics of health promotion programs (OMS, 2009), the definition and selection of a community is a key issue. In our research in the Region of Brussels a geographical approach towards community has been privileged, choosing the neighborhood - the space where daily life activities take place and to which inhabitants identify themselves (IBSA, 2007) - as the scale of analysis. This decision is motivated by several reasons. On the one hand, the key principles of intersectionality (Bowleg, 2012 ; Christensen et Qvotrup, 2012) - especially the mutual constitution of social categories and the idea that subjectivities are complex, intersecting and context-dependent - make the selection of categories for the intersectional analysis an "empirical open question" (Hancock, 2007, p. 251). Instead of selecting a particular group of the population based on a supposed common identity (i.e. 'women', 'migrants'), we propose to analyze all the social categories that emerge from the whole population of a neighborhood (Christensen et Qvotrup, 2012). This strategy would prevent a 'minoritising approach' that risks the homogenization and stigmatization of certain groups (Giolla Easpaig et al., 2013).

On the other hand health-related practices take place in particular contexts with symbolic and material dimensions. In our research the neighborhood is considered an element of these practices as it offers material resources and a differential access to them for different social categories present in this territory. A third set of reasons is the fact that participatory processes for community health promotion cannot be implemented in geographical spaces that are too big or too populous (Marchioni, 1999) and that behavioral change programs on a large scale often fail (Frohlich et Abel, 2014).

Beyond an epidemiological perspective that focuses on the description and quantification of social inequalities of health, our research pays attention to the processes that lead to those 
inequalities. Instead of considering the individuals as being determined by the social systems, we suggest the adoption of a poststructural approach that conceives the relation between social categories and subjects as a bidirectional proccess (Staunaes, 2003) in order to overcome the traditional tension 'structure-agency'. Drawing on Foucault's notion of subjectification and Butler's notion of performativity we define health-related practices as social practices that are influenced by the imbrication of social categories and at the same time contribute to their construction. That is, social categories are not what a subject is, but what a subject perform. We suggest an interactionist approach that takes into consideration not only the symbolic aspects of subjectivities but also the material ones. In particular we draw the attention to the role of the body, of individual ressources and of the ressources of the neighborhood as important elements of health-related practices.

Together with this theoretical perspective we present a methodological proposal that attempts to overcome the methodological challenges raised by the intersectional approach that could inspire empirically grounded research in the health domain.

In this regard, we suggest the group analysis method (Van Campenhoudt et al, 2009) as a useful tool to identify relevant health-related practices in the selected neighborhood(s). This technique allows the diversity of experiences of the participants to be considered and the most relevant social categories for the intersectional analysis to be identified. As in relation to the analysis of intersectionality itself, we propose the technique of the narratives as being able to capture the intersection of social categories as it is lived and experienced by the subjects (Christensen and Qvotrup Jensen, 2012). The topics of the narratives are based on the health-related practices identified in the previous phase. Finally, we propose a comparative approach - both within neighborhoods and inside neighborhoods - to better understand the processes that lead people to different health-related practices.

To conclude, we draw attention to the potentialities that the incorporation of the intersectional approach to health promotion could offer, such as understanding health-related practices in all their complexity, understanding the link between social inequalities and health inequalities, the relation between the neighborhood, health and gender and the possible conception of programs that address the complexity of the population.

\section{INDEX}

Mots-clés : genre, intersectionnalité, inégalités sociales et territoriales de santé, jeunes, promotion de la santé, quartiers

\section{AUTEUR}

\section{SARA AGUIRRE SÁNCHEZ-BEATO}

Doctorante, Université libre de Bruxelles - ULB, Belgique 